\title{
An electrically driven polariton laser
}

\author{
A. Rahimi-Iman ${ }^{1}$, C. Schneider ${ }^{1}$, N. Y. Kim ${ }^{2,3}$, J. Fischer ${ }^{1}$ I. G. Savenko ${ }^{4,5}$, M. Amthor ${ }^{1}$, \\ L. Worschech ${ }^{1}$, V. D. Kulakovskii ${ }^{6}$, I. A. Shelykh ${ }^{4,5}$, M. Kamp ${ }^{1}$, S. Reitzenstein ${ }^{1,7}$,

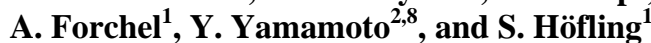 \\ 1) Technische Physik, Physikalisches Institut and Wilhelm Conrad Röntgen Research Center for Complex Material Systems, \\ Universität Würzburg, Am Hubland, D-97074 Würzburg, Germany \\ 2) E. L. Ginzton Laboratory, Stanford University, Stanford CA, 94305, USA. \\ 3) Institute of Industrial Science, University of Tokyo, 4-6-1 Komaba, Meguro-ku, Tokyo 153-8505, Japan. \\ 4) Science Institute, University of Iceland, Dunhaga 3, IS-107, Reykjavik, Iceland. \\ 5) Division of Physics and Applied Physics, Nanyang Technological University 637371, Singapore. \\ 6) Institute of Solid State Physics, Russian Academy of Science, 142432, Chernogolovka, Russia \\ 7) Institut für Festkörperphysik, Technische Universität Berlin, D-10623 Berlin, Germany. \\ 8) National Institute of Informatics, Hitotsubashi, Chiyoda-ku, Tokyo 101-8430, Japan. \\ arash.rahimi-iman@physik.uni-wuerzburg.de
}

\begin{abstract}
Polariton lasing under electrical pumping is observed in a GaAs multi-quantum-well microcavity diode. Lasing in the strong-coupling regime is unambiguously evidenced by detection of Zeeman-split emission in an external magnetic field as a result of the polaritons' excitonic content.

OCIS codes: (140.7270) Vertical emitting lasers; (140.3948) Microcavity devices; (140.5960) Semiconductor lasers
\end{abstract}

\section{Introduction}

While conventional lasers are based on stimulated emission which requires a certain amount of power for population inversion, a new type of coherent light source named polariton laser uses stimulated scattering into one energy state, from which polaritons decay spontaneously with radiation features similar to that of a conventional photonic laser source. Polaritons are formed in quantum well (QW) microcavities as the consequence of strong exciton-photon coupling. Above a critical particle density, the bosonic polariton gas can undergo a condensation process [1, 2]. This promises a more energy-efficient laser operation in terms of lower threshold powers. In this work, a polariton laser under electrical pumping is presented [3], for which strong light-matter coupling is unambiguously evidenced due to a Zeeman-splitting of the laser mode when an external magnetic field is applied [4]. This provides access to a practical use of polaritonic light sources for future opto-electronic applications.

\section{Experimental results}

Electroluminescence from a four InGaAs QW microcavity (p-i-n) diode based on 20- $\mu$ m micropillars etched into the cavity wafer is measured via far-field resolved spectroscopy. The structure consists of doped AlAs/GaAs distributed Bragg reflectors (DBRs) and an intrinsic cavity, allowing for current injection. Current-density-dependent investigations of the diodes reveal a two threshold behavior, with energy-momentum dispersions showing three emission regimes. These are attributed to low-density lower-energy-branch polariton emission, polariton lasing and cavity mediated photon lasing in the weak-coupling regime. A spin-split polariton ground-state emission is observed in polarization-resolved spectroscopy up to a current density corresponding to the photon-lasing threshold under the presence of an external magnetic field. At $5 \mathrm{~T}$, the dependency of the detected Zeeman-splitting on the current density demonstrates a clear transition from strong coupling above a first threshold towards a weakly coupled cavity mode, providing clear proof of polariton laser operation between the two observed thresholds.

\section{References}

[1] H. Deng et al, “Condensation of Semiconductor Microcavity Exciton Polaritons”, Science 298, 199 (2002).

[2] J. Kasprzak et al, “Bose-Einstein-Condensation of Polaritons”, Nature 443, 409 (2006).

[3] C. Schneider, A. Rahimi-Iman et al, „An electrically pumped polariton laser“, Nature 497, 348 (2013).

[4] A.V. Larionov et al, "Polarized Nonequilibrium Bose-Einstein Condensates of Spinor Exciton-Polaritons in a Magnetic Field”, Phys. Rev. Lett. 105, 256401 (2010) 